\title{
ESTIMATION DES CAPTURES EFFECTUÉES DURANT LA SAISON 1991-1992 PAR LES PÊCHEURS À LA LIGNE DANS UN GRAND COURS D'EAU FRANÇAIS (LA SAÔNE).
}

\section{T. ChANGEUX *}

Direction Régionale de l'Environnement Rhône-Alpes, 19 rue de la Villette, 69425 Lyon Cedex 03, France.

\section{RÉSUMÉ}

Une enquête de terrain et une enquête téléphonique ont été réalisées durant les années 1991 et 1992, auprès de deux grands échantillons de pêcheurs à la ligne de la Saône, afin d'établir leur nombre d'heures de pêche annuel et le poids horaire de leurs captures. La prise en compte des valeurs nulles, et l'ajustement des valeurs non nulles au modèle log-normal, donnent un temps de pêche annuel voisin de $136 \mathrm{~h} / \mathrm{an}$ et un poids horaire de capture de $152 \mathrm{~g} / \mathrm{h}$. Le nombre important des pêcheurs de la Saône (estimé à 45600 ) conduit à la capture annuelle de 950 tonnes de poissons, soit $3,16 \mathrm{t} / \mathrm{km} / \mathrm{an}$ ou $183 \mathrm{~kg} / \mathrm{ha} / \mathrm{an}$. Ces valeurs se rapprochent de la productivité piscicole théorique de la rivière.

Mots-clés : pêche sportive, capture, effort, pêcheur à la ligne, distribution log-normale, grand cours d'eau.

\section{ANGLERS' CATCHES ASSESSMENT DURING THE SEASON 1991-1992 IN A FRENCH LARGE RIVER (THE SAÔNE).}

\begin{abstract}
A field survey and a telephone survey were carried out during the years 1991 and 1992, through two large samples of sport anglers fishing in the River Saône, in order to determine both their annual time of fishing and their catches weight per hour. Taking into account the zero values, and adjusting the non-zero values to a log-normal model, give a time of fishing close to $136 \mathrm{~h} /$ year and a catch weight per hour of $152 \mathrm{~g} / \mathrm{h}$. The numerous anglers of the River Saone (estimated to 45600 ) lead to an annual catch of 950 tons of fishes, corresponding to $3.16 \mathrm{t} / \mathrm{km} /$ year or $183 \mathrm{~kg} / \mathrm{ha} /$ year. These values are close to the theoretical fish production of the river.
\end{abstract}

Key-words : sport fishery, catch, effort, angler, log-normal distribution, large river.

\section{INTRODUCTION ET OBJECTIFS}

En France, la pêche des eaux douces est surtout une activité de loisir. Les pêcheurs à la ligne sont environ 3 millions à pêcher au moins 5 jours par an (ANONYME, 1992). Ils sont

- Adresse actuelle : Conseil Supérieur de la Pêche, Direction Générale, 134 avenue de Malakoff, 75116 Paris, France. 
nombreux à fréquenter les petites rivières à la recherche de truites ou bien les étangs et les grands cours d'eau pour la pêche des carnassiers'.

Dans ce contexte, la Saône se présente comme un des rares grands cours d'eau où la pêche des poissons résidents est importante. La fédération de Saône-et-Loire rassemble un nombre considérable de pêcheurs à la ligne (deuxième fédération de pêche de France par le nombre de taxes ordinaires perçues, selon ANONYME, 1993). En outre, ils sont souvent spécialisés dans la pêche des eaux de seconde catégorie.

Par ailleurs, les pêcheurs aux engins du bassin du Rhône se concentrent également sur la Saône aval (CHANGEUX et ZYLBERBLAT, 1994a). La ressource piscicole de la rivière est donc particulièrement convoitée et son partage entre les différentes catégories de pêcheurs reste difficile en l'absence de statistiques sur les captures des pêcheurs à la ligne.

Notre objectif est ici d'évaluer les captures effectuées annuellement par ces pêcheurs dans la partie de la Saône traversant les départements du Rhône, de l'Ain, de la Saône-et-Loire et de la Côte-d'Or, soit un linéaire total de $301 \mathrm{~km}$ de rivière, estimé à 5200 ha à partir des cartes au 1/50000ème du "Schéma de vocation piscicole " (DBRMC, 1994b).

L'estimation du nombre total de pêcheurs à la ligne de la Saône et leur répartition spatiotemporelle ayant été abordées plus particulièrement par les études du LATEC (1993), sur la base des mêmes données, nous nous contenterons de rappeler ici les résultats utilisés par la suite :

- un nombre total de pêcheurs évalué à 45600 , répartis inégalement sur le linéaire ;

- une variation cyclique de la fréquentation des pêcheurs et des espèces qu'ils capturent, avec une forte tendance saisonnière.

\section{MATÉRIEL ET MÉTHODES}

\subsection{Protocole d'échantillonnage}

Le nombre total de pêcheurs étant connu, l'estimation des captures totales (toutes espèces confondues) peut être déduite simplement:

- du temps moyen passé par le pêcheur à exercer sur la Saône ;

- du poids moyen des captures effectuées pendant ce temps.

Chacun de ces deux paramètres a été estimé indépendamment à partir de deux échantillons distincts où chaque pêcheur constitue un élément tiré au hasard.

Le premier échantillon a été contacté par téléphone entre juillet 1991 et juillet 1992 (inclus). Les adresses et les numéros de téléphone des pêcheurs ont été extraits des carnets à souches des AAPPMA riveraines pour l'année en cours. Chaque mois, il a été procédé à environ 60 entretiens téléphoniques, au cours desquels il était demandé au pêcheur le nombre d'heures passées sur la Saône au cours des trois mois précédents. L'échantillon de pêcheurs a été établi en tenant compte de l'effectif de chaque association (stratification de l'échantillon), de manière à être le plus représentatif possible de l'ensemble des pêcheurs de Saône. Un total de 602 durées trimestrielles de pêche a été collecté.

Pour obtenir les captures des pêcheurs, des enquêtes de terrain ont été organisées avec les gardes-pêche des fédérations départementales concernées, de mai 1991 à mai 1992 (inclus) ; l'unité de temps retenue étant l'heure passée avant la visite sur le terrain. Le garde notait l'espèce, la taille et le nombre de poissons pêchés par chaque pêcheur contrôlé pendant ce laps de temps.

Un total de 1003 pêcheurs a été contrôlé. Comme ces tournées avaient également comme objectif de déterminer les variations de fréquentation au bord de la rivière (LATEC, 1993), elles ont été réparties équitablement dans l'espace et dans le temps. Le poids horaire des captures 
a été ensuite déduit des courbes taille-poids de chaque espèce en usage au Laboratoire d'écologie des systèmes fluviaux (Arles).

Même si les deux périodes d'enquêtes ne correspondent pas exactement, le cycle annuel d'exploitation a été bouclé avec un nombre légèrement plus important de pêcheurs durant les mois d'été pour tenir compte d'une fréquentation plus importante en cette saison.

\subsection{Variations des paramètres à estimer}

Le nombre d'heures de pêche trimestriel comprend beaucoup de valeurs nulles ( 371 pêcheurs sur 602). Une fois éliminées ces valeurs, la variable présente une importante queue de distribution (figure 1a), car un petit nombre de pêcheurs se distinguent par une fréquentation plus assidue de la rivière. Malgré leur faible effectif, ils constituent une importante composante de l'effort de pêche.

Ainsi, qu'il s'agisse des données brutes, ou bien après élimination des valeurs nulles, la distribution dissymétrique du temps trimestriel de pêche ne peut pas être positionnée par une valeur centrale comme la moyenne.

En revanche, après transformation par les logarithmes népériens (In) des données non nulles (figure 1b), la distribution se rapproche de la normalité (test de Lilliefors significatif à $5 \%$ seulement). Elle peut alors être positionnée par sa moyenne $(m=3,719)$ et sa variance $\left(s^{2}=1,535\right)$ peut être retenue comme paramètre de dispersion.

L'étude des captures horaires montre que cette variable présente les mêmes caractéristiques que le nombre d'heures de pêche trimestriel. Sur 1003 pêcheurs, 498 sont bredouilles et, une fois retirées les prises nulles, la variable transformée suit une loi normale de paramètres $m=4,977$ et $s^{2}=1,472$ (test de Lilliefors non significatif).

Ainsi, les distributions obtenues peuvent se séparer en deux parties : une partie où la variable s'annule, et une partie où elle suit une loi log-normale. Cela confirme les résultats déjà établis sur d'autres pêcheurs à la ligne (GERARD, 1993 ; CRYER et MACLEAN, 1991 ; DBRMC, 1990) et peut être certainement généralisé à la plupart des captures par unité d'effort telles qu'elles sont fournies par cette catégorie de pêcheurs.

\subsection{Principe du calcul}

Les deux paramètres à estimer peuvent être définis comme des variables aléatoires $Z$ telles que :

$\left\{\begin{array}{l}P(Z=0)=w \\ P(Z \neq 0)=(1-w) \\ \text { et } \\ L \text { densitéde probabilité de } Z \text { sachant } Z \neq 0 \\ \text { suit une loi log - normale }\end{array}\right.$ avec $w=\frac{n b \text { de réalisations de } Z=0}{n b \text { de réalisations de } Z}$

La moyenne de $Z$ s'écrit $E(Z)=(1-w) E(L)$, et sa variance :

$$
\begin{aligned}
& V(Z)=E\left(Z^{2}\right)-(E(Z))^{2}=(1-w) E\left(L^{2}\right)-(1-w)^{2}(E(L))^{2} \\
& V(Z)=(1-w)\left[E\left(L^{2}\right)-(1-w)(E(L))^{2}\right] \\
& V(Z)=(1-w)\left[E\left(L^{2}\right)-(E(L))^{2}+w(E(L))^{2}\right] \\
& V(Z)=(1-w) V(L)+w(1-w)(E(L))^{2}
\end{aligned}
$$



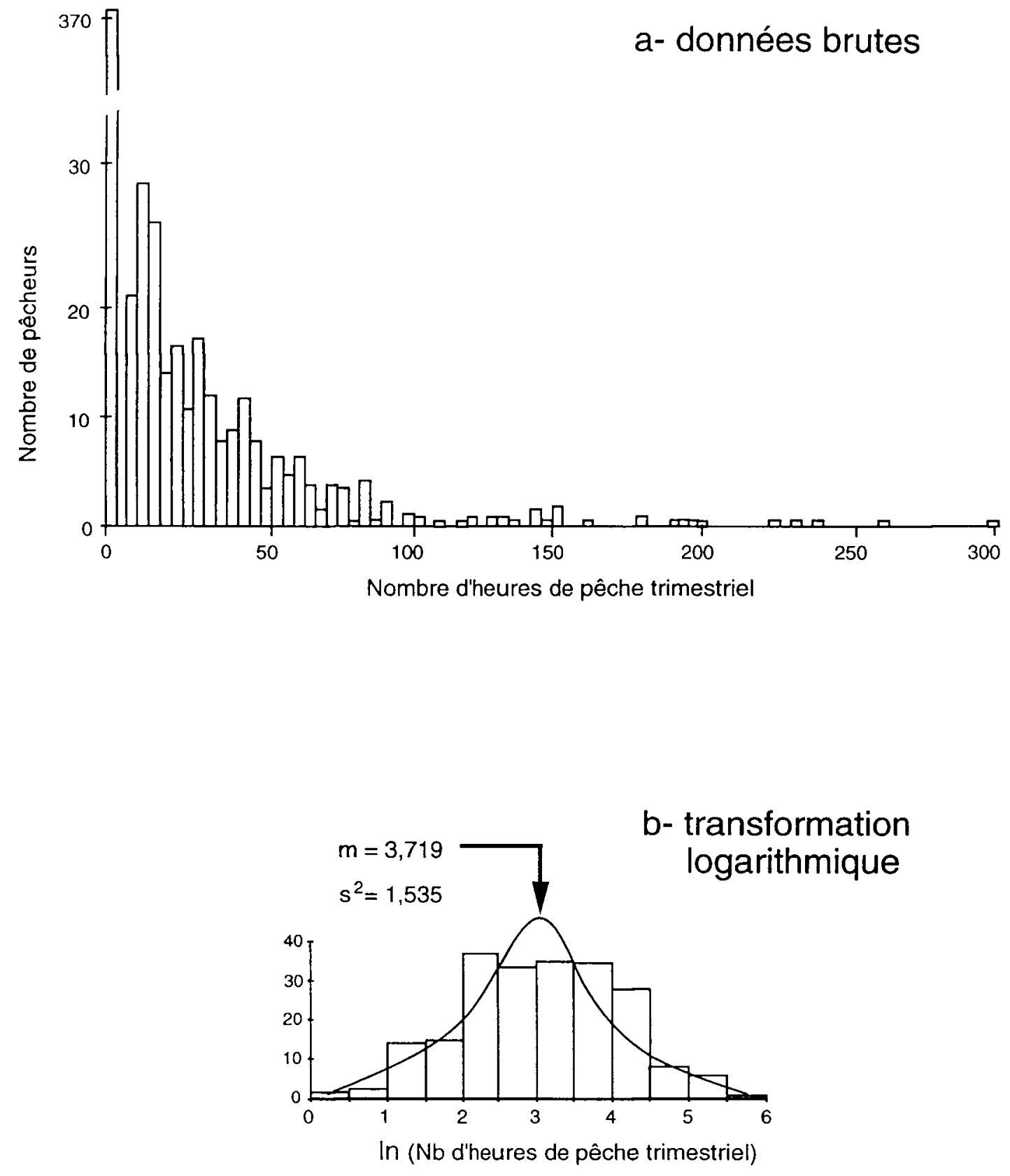

Figure 1 : Distribution du temps de pêche trimestriel (h/3 mois). a : données brutes, montrant de nombreuses valeurs nulles et une importante dissymétrie ; $b$ : données après transformation des valeurs non nulles par les logarithmes népériens (In), montrant que la distribution est log-normale et peut être caractérisée par sa moyenne $(\mathrm{m})$ et sa variance $\left(s^{2}\right)$.

Figure 1 : Distribution of the quarterly fishing time (number of hours per 3 months). a : raw data, showing the numerous zero values and an important asymmetry ; $b$ : data after transformation of non-zero values by neperian logarithms (In), showing that the distribution is following a log-normal model and can be characterized by its mean (m) and variance $\left(\mathrm{s}^{2}\right)$. 
Dire que $L$ suit une loi log-normale revient à dire que :

$$
\ln (L)=X \text { tend vers une Loi Normale }\left(m, \sigma^{2}\right) \text {. }
$$

Dans ce cas, DAGNÉLIE (1992) donne pour moyenne et variance :

$$
E(L)=e^{m+\frac{\sigma^{2}}{2}} \text { et } V(L)=e^{2 m+\sigma^{2}}\left(e^{\sigma^{2}}-1\right)
$$

Si on pose $\alpha=m+\frac{\sigma^{2}}{2}$, en remplaçant $E(L)$ et $V(L)$ dans les équations précédentes, on obtient :

$$
\begin{aligned}
& E(Z)=(1-w) e^{\alpha} \\
& V(Z)=(1-w) e^{2 \alpha}\left[e^{\sigma^{2}}+w-1\right]
\end{aligned}
$$

Le domaine de confiance à $95 \%$ de part et d'autre de la valeur estimée est égal à 1,96 fois l'écart type de la moyenne :

$$
\text { Ecart Type }(\bar{Z})=\sqrt{\frac{V(\bar{Z})}{n}} \text { avec } n=n b \text { de réalisations de } Z \text {. }
$$

Le coefficient de variation de l'estimation est défini par le rapport:

$$
\mathrm{CV}(\overline{\mathrm{Z}})=\frac{\text { Ecart Type }(\overline{\mathrm{Z}})}{\bar{Z}}
$$

\section{RÉSULTATS}

\subsection{Estimation du temps de pêche annuel}

Nous avons vu que $m=3,719$ et $s^{2}=1,535$ donc en remplaçant la valeur théorique $\sigma$ par la valeur observée $s, \alpha=4,4865$.

De plus, $w=371 / 602$, donc la moyenne et la variance du temps trimestriel de pêche sont respectivement $E(Z)=34,4$ et $V(Z)=13002,8$. Ce qui donne :

$$
\text { Ecart Type }(\bar{Z})=\sqrt{\frac{13002,8}{602}}=4,648
$$

Soit un domaine de confiance à $95 \%$ égal à $34,1 \pm(1,96 \bullet 4,648)$, ou un intervalle de [25-44] h/3 mois et un coefficient de variation de $13,6 \%$.

Ces valeurs sont à multiplier par quatre pour obtenir le temps de pêche annuel du pêcheur de Saône, qui se situe donc à 95 \% dans l'intervalle 136,4 (1,96 • 18,592), soit [101-174] h/an.

\subsection{Estimation de la prise horaire}

En suivant le même calcul que précédemment, nous obtenons $E(Z)=152,5$ à partir des valeurs de $w=498 / 1003, m=4,977$ et $s^{2}=1,472$.

On obtient également $V(Z)=177910$. Ce qui donne :

$$
\text { Ecart Type }(\bar{Z})=\sqrt{\frac{177910}{1003}}=13,32
$$

Les prises horaires d'un pêcheur de Saône se situent donc à $95 \%$ dans l'intervalle $152,5 \pm(1,96 \cdot 13,32)$, soit $[126-179] \mathrm{g} / \mathrm{h}$ et un coefficient de variation de $8,7 \%$. 


\subsection{Estimation de la prise annuelle}

Les prises horaires et les temps de pêche estimés précédemment nous permettent d'indiquer que chaque pêcheur prend en moyenne 152,5 $1136,4=20801 \mathrm{~g} / \mathrm{an}$, soit 20,8 $\mathrm{kg} /$ pêcheur/an.

Sans tenir compte des incertitudes sur le nombre total de pêcheurs de Saône, absent de l'étude du LATEC (1993), la quantité totale prélevée par les pêcheurs à la ligne est donc de $20,8 \cdot 45600=948480 \mathrm{~kg} / \mathrm{an}$, soit environ $950 \mathrm{t} / \mathrm{an}$.

Compte tenu des incertitudes sur le poids horaire et la durée annuelle de pêche, ce chiffre est en réalité compris à $95 \%$ entre $101 \cdot 126 \cdot 45600=580 \cdot 10^{6} \mathrm{~g} / \mathrm{an}$ et $174 \cdot 179 \cdot 45600=1420 \cdot 10^{6} \mathrm{~g} / \mathrm{an}$.

\section{DISCUSSION-CONCLUSION}

Avec $152 \mathrm{~g} / \mathrm{h}$, les captures horaires du pêcheur de Saône sont du même ordre de grandeur que celles des pêcheurs d'autres cours d'eau comparables ( $250 \mathrm{~g} / \mathrm{h}$ sur le Rhône, selon DBRMC (1990) ; voir également les résultats de COWX et al. (1986) et de COWX (1991) sur les cours inférieurs de la Derwent et de la Trent, en Angleterre).

Pour connaître avec encore plus de précision l'impact des pêcheurs à la ligne sur les stocks, il serait intéressant d'envisager la part des prises remises à l'eau et leurs possibilités de survie, comme le fait GERARD (1993) dans son étude de deux canaux belges. Cette proportion risque d'être importante pour les espèces peu consommées, comme les grands cyprinidés (carpes, brèmes, gardons, chevaines...) qui sont abondants dans le peuplement (LESF, 1993). Néanmoins, il faut rappeler ici que les pêcheurs de Saône recherchent en priorité le sandre pour leur consommation. Or, cette espèce représente environ $40 \%$ des prises durant la saison étudiée (DBRMC, 1994a). Le risque d'une remise à l'eau importante des captures est donc minime par rapport à ce qu'observe GERARD (1993) pour le gardon.

Le nombre d'heures par an et par pêcheur semble raisonnable. Une pêche aussi spécialisée que celle de la truite de mer dans les rivières du nord-ouest de la France atteint déjà $84 \mathrm{~h} /$ pêcheur/an (CSP, 1993). Une enquête menée en 1983 indique que $70 \%$ des pêcheurs français pratiquent plus de 30 jours par an (ANONYME, 1992). Cela représente plus de $120 \mathrm{~h} / \mathrm{an}$ si l'on fixe la journée de pêche à 4 heures. Toutefois, le temps de pêche annuel serait en baisse selon les dernières données disponibles. Quoi qu'il en soit, le pêcheur de Saône, avec $136 \mathrm{~h} / \mathrm{an}$, reste donc particulièrement assidu.

A partir des chiffres qui viennent d'être énoncés, il apparaît que les captures par pêche à la ligne représentent environ 9 fois plus que les prélèvements par pêche aux engins, sur l'ensemble du cours d'eau, estimés en moyenne à 106 tonnes (CHANGEUX et ZYLBERBLAT, 1994b). Toutefois, ce dernier chiffre concerne les années 1988 et 1989, alors que durant la saison 1991-1992, lors de notre enquête, le stock de sandres était à son niveau le plus haut depuis 1988 (LESF, 1993). Ainsi, les captures des pêcheurs à la ligne, qui se rapportent à cette saison particulière, sont certainement plus importantes qu'en temps normal.

Une fois rapportées à la surface de rivière étudiée ( $5200 \mathrm{ha}$ ), les 950 tonnes de captures représentent $183 \mathrm{~kg} / \mathrm{ha} / \mathrm{an}$. Cette valeur est comprise entre les 178 et les $241 \mathrm{~kg} / \mathrm{ha} / \mathrm{an}$ obtenus par GERARD (1993; p. 111) pour les captures (brèmes et gardons confondus) effectuées par pêche sur l'ancien canal Charleroi-Bruxelles à Feluy et sur le canal Ath-Blaton à Blaton.

En termes de prélèvements, nos chiffres sont situés dans les valeurs moyennes de productivité en poissons avancées pour les cours d'eau des zones tempérées (WELCOMME, 1985). Les 3,16 tonnes $/ \mathrm{km}$ obtenues en rapportant les 950 tonnes au linéaire étudié $(301 \mathrm{~km})$ se rapprochent de la productivité théorique de LÉGER (1945), estimée à 3,2 tonnes $/ \mathrm{km}$ dans la partie inférieure du cours de la rivière. 
II est donc certain que les captures opérées par les pêcheurs à la ligne sont importantes, au regard des possibilités théoriques de production. Mais il faut signaler que ces dernières n'ont pas été réévaluées depuis les années quarante, et ne prennent donc pas en compte les modifications ultérieures (augmentation des concentrations en sels nutritifs, modifications des berges et du cours, etc.). De plus, elles ne fournissent qu'un ordre de grandeur, sans tenir compte des variations interannuelles. Ainsi, pour connaître avec précision l'impact de la pêche à la ligne, il conviendrait de reproduire ce type d'étude sur plusieurs années.

Du point de vue méthodologique, les coefficients de variation obtenus ici sur les différents paramètres estimés sont semblables à ceux rapportés par GERARD (1993) pour d'autres études comparables. Ils conduisent à une précision suffisante de l'estimation des captures totales. Toutefois, un ajustement de l'effort d'échantillonnage suivant les variations de fréquentation observées dans l'espace et dans le temps (DBRMC, 1994a; LATEC, 1993), permettrait d'avoir à l'avenir la même précision pour un moindre effort d'échantillonnage.

Enfin, lorsqu'il ne peut pas être clairement démontré que les valeurs non-nulles suivent une loi log-normale, MYERS et PEPIN (1990) considèrent que la moyenne et la variance de l'échantillon sont des estimateurs suffisamment robustes. II reste que la précision des estimations globales, obtenues par extrapolation, est alors nettement moins bonne.

\section{REMERCIEMENTS}

Ce travail s'intègre dans le Schéma Interdépartemental de Vocation Piscicole de la Saône dont la mise en oeuvre a été confiée à la Direction Régionale de l'Environnement Rhône-Alpes, avec comme maître d'ouvrage les Fédérations Départementales des AAPPMA du Rhône, de l'Ain, de la Saône-et-Loire et de la Côte-d'Or.

Claire SANLAVILLE-BOISSON (Laboratoire de Biologie Micromoléculaire et Phytochimie, Université $\mathrm{Cl}$. Bernard Lyon 1) a effectué les manipulations sous base de données. Pierre-Marie COMBE (Laboratoire d'Analyse et de Techniques Economiques, Université de Bourgogne, Dijon) et son équipe ont effectué les enquêtes téléphoniques. Les gardes-pêche employés dans les fédérations concernées ont effectué les enquêtes de terrain. Daniel CHESSEL (Laboratoire d'Ecologie des Eaux douces et des Grands Fleuves, Université Cl. Bernard Lyon 1) a calculé les estimations.

\section{BIBLIOGRAPHIE}

ANONYME, 1992. Qui sont les pêcheurs ? La Gazette Officielle de la Pêche et de l'Eau, 1074, 714.

ANONYME, 1993. Toutes les données de l'évolution du nombre de pêcheurs entre 1991 et 1992. La Gazette Officielle de la Pêche et de l'Eau, 1111, 2-3.

CHANGEUX T., ZYLBERBLAT M., 1994a. Analyse des statistiques de pêche aux engins dans le bassin du Rhône. Première partie : étude de l'effort de pêche. Bull. Fr. Pêche Piscic., 330, 245-269.

CHANGEUX T., ZYLBERBLAT M., 1994b. Analyse des statistiques de pêche aux engins dans le bassin du Rhône. Seconde partie : étude des captures. Bull. Fr. Pêche Piscic., 330, 271294.

COWX I.G., 1991. The use of angler catch data to examine potential fishery management problems in the lower reaches of the River Trent, England in COWX I.G., Catch Effort Sampling Strategies, 154-165, Fishing News Books, Blackwell Scientific Publ., Oxford (Royaume-Uni).

COWX I.G., FISHER K.A.M., BROUGHTON N.M., 1986. The use of anglers' catches to monitor fish population in large water bodies, with particular reference to the River Derwent, Derbyshire, England. Aquaculture and Fisheries management, 17, 95-103. 
CRYER M., MACLEAN G.D., 1991. Catch effort in a New Zealand recreational trout fishery, a model and implication for survey design in COWXI.G. Catch Effort Sampling Strategies, 61-71, Fishing News Books, Blackwell Scientific Publ.. Oxford (Royaume-Uni).

CSP (Conseil Supérieur de la Pêche), 1993. La truite de mer dans le nord-ouest, année 1992. Rapport du CSP, Paris, mars 1993, 41 p. + annexes.

DAGNÉLIE P., 1992. Statistique théorique et appliquée, volume 1. Presses agronomiques de Gembloux, Gembloux (Belgique), 492 p.

DBRMC (Délégation de Bassin Rhône-Méditerranée-Corse), 1990. La pêche aux lignes sur le Rhône. Schéma de vocation piscicole du fleuve Rhône, Document $n^{\circ} 6$, Direction Régionale de l'Environnement Rhône-Alpes, Lyon (France), $10 \mathrm{p}$.

DBRMC (Délégation de Bassin Rhône-Méditerranée-Corse), 1994a. Les captures par pêche à la ligne dans la Saône. Schéma de vocation piscicole de la Saône, Document technique $\mathrm{n}^{\circ} 2$, "Les poissons, étude du peuplement piscicole, des stocks exploités et de l'état sanitaire du poisson", Direction Régionale de l'Environnement Rhône-Alpes, Lyon (France), 10 p. + annexes.

DBRMC (Délégation de Bassin Rhône-Méditerranée-Corse), 1994b. Cartes au 1/50000ème. Schéma de vocation piscicole de la Saône, Direction Régionale de l'Environnement Rhône-Alpes, Lyon (France).

GERARD P., 1993. Communauté piscicole de deux canaux et évolution de l'impact de la pêche récréative sur la dynamique de population de gardon Rutilus rutilus (L.). Mémoire de doctorat en sciences agronomiques, Université catholique de Louvain, 196 p.

LATEC (Laboratoire d'Analyse et de Techniques Economiques, Dijon), 1993. La Saône et ses pêcheurs, état actuel de la pêche aux lignes, propositions d'action pour les partenaires socio-économiques. Schéma de vocation piscicole de la Saône, Document technique $\mathrm{n}^{\circ} 3$, «Les pêcheurs, étude socio-économique de la pêche amateur aux lignes et de la pêche professionnelle aux engins", DIREN Rhône-Alpes, Lyon, 40 p.

LÉGER L., 1945. Carte piscicole du département du Rhône. Travaux du Laboratoire d'Hydrobiologie Piscicole, Université de Grenoble.

LESF (Laboratoire d'Écologie des Systèmes Fluviaux, Arles), 1993. Etude du peuplement piscicole et de la pêche amateur et professionnelle aux engins. Schéma de vocation piscicole de la Saône, Document technique $n^{\circ} 2$, "Les poissons, étude du peuplement piscicole, des stocks exploités et de l'état sanitaire du poisson", Direction Régionale de l'Environnement Rhône-Alpes, Lyon (France), 110 p. + annexes.

MYERS R.A., PEPIN P., 1990. The robustness of lognormal-based estimators of abundance. Biometrics, 46, 1185-1192.

WELCOMME R.L., 1985. River fisheries. FAO Fish. Tech. Pap. n`262, Rome, 330 p. 